\title{
Synthesis of char-based acidic catalyst for methanolysis of waste cooking oil: an insight into a possible valorization pathway for the solid by-product of gasification
}

\begin{abstract}
Gasification-based char from a commercial small-scale gasification plant was converted into an acidic catalyst for methanolysis of waste cooking oil (WCO). The char-based acidic catalyst was synthesized by the sulfonation of gasification char with sulfuric acid. Functional groups, acid density, morphological and surface properties were characterized and measured by using Fourier transform infrared red spectroscopy, ammonia temperature programmed desorption, field emission scanning electron microscope and Brunauer-Emmett-Teller, respectively. Wood char based acid catalyst showed higher surface area of $337 \mathrm{~m} 2 / \mathrm{g}$ and acid density of $2.94 \mathrm{mmol} / \mathrm{g}$. The reaction variables such as methanol/oil molar ratio, catalyst loading, reaction time and temperature, were studied. The optimum reaction conditions, 9:1 methanol/oil ratio, $6 \mathrm{wt} \%$ catalyst loadincxg, $130 \mathrm{~min}$ reaction time at $65{ }^{\circ} \mathrm{C}$, gave $96 \%$ of ester conversion. The recovered catalyst was washed and reused without any activation (calcination), still giving $81 \%$ of ester conversion after five reaction cycles. Furthermore, fuel properties of WCO methyl esters were determined as per the ASTM and EN biodiesel standards. The utilization of char from biomass gasification for the synthesis of an acid catalyst for biodiesel production allows achieving a twofold objective. On the one side, the valorization of a material which is presently wasted. On the other side, the production of a catalyst that can effectively convert WCO into biodiesel in a single step process, therefore allowing a simplified procedure and lower operational temperatures. In addition, the obtained catalyst showed an interesting efficiency in decreasing free fatty acid of WCO and a considerable recyclability of the catalyst.
\end{abstract}

Keyword: Gasification-based char; Sulfonation; Reaction condition optimization; Reusability; Fuel properties 